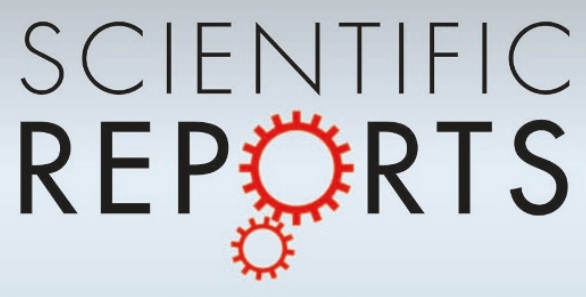

OPEN

SUBJECT AREAS:

IMAGING AND SENSING

IMAGING TECHNIQUES

TOTAL INTERNAL REFLECTION MICROSCOPY

OPTICAL IMAGING

Received

14 May 2013

Accepted

13 June 2013

Published

4 July 2013

Correspondence and requests for materials should be addressed to S.R. (Srinivasan@eng. ucsd.edu) or R.L. (rlal@

ucsd.edu)

* These authors contributed equally to this work.

$\uparrow$ Current address: Materials Research Science and Engineering Center, Northwestern University, Evanston IL 60208.

\section{High performance, LED powered, waveguide based total internal reflection microscopy}

\author{
Srinivasan Ramachandran ${ }^{1 *}$, Daniel A. Cohen ${ }^{2 *}$, Arjan P. Quist ${ }^{3 * \dagger}$ \& Ratnesh Lal
}

\begin{abstract}
'University of California San Diego, Department of Mechanical and Aerospace Engineering and Department of Bioengineering, La Jolla, CA 92093, ${ }^{2}$ University of California Santa Barbara, Department of Materials, Santa Barbara CA $93106,{ }^{3}$ RC Nano Corporation, 2210 Midwest Road, Oak Brook, IL 60523, USA.
\end{abstract}

Total internal reflection fluorescence (TIRF) microscopy is a rapidly expanding optical technique with excellent surface sensitivity and limited background fluorescence. Commercially available TIRF systems are either objective based that employ expensive special high numerical aperture (NA) objectives or prism based that restrict integrating other modalities of investigation for structure-function analysis. Both techniques result in uneven illumination of the field of view and require training and experience in optics. Here we describe a novel, inexpensive, LED powered, waveguide based TIRF system that could be used as an add-on module to any standard fluorescence microscope even with low NA objectives. This system requires no alignment, illuminates the entire field evenly, and allows switching between epifluorescence/TIRF/bright field modes without adjustments or objective replacements. The simple design allows integration with other imaging systems, including atomic force microscopy (AFM), for probing complex biological systems at their native nanoscale regimes.

nterfacial and near-surface processes are critical components for physical as well as biological systems, yet our understanding of these processes is limited by the lack of simple and robust imaging techniques. For example, the cell plasma membrane and its adjoining cytoplasmic and extracellular matrix regions (collectively referred to as the membrane zone) are among the most dynamic regions of a cell. Various critical processes like cell adhesion, cell signaling and membrane trafficking occur at this membrane zone. It is crucial to understand the molecular mechanisms of the above processes to comprehend health and disease. Imaging these dynamic zones with conventional optical techniques remains a challenge due to the large depth of field of the microscope objectives that collect out of focus light (background noise) that obscures the informative signals, limiting the resolution of cellular events across the membrane zone.

Total internal reflection fluorescence microscopy (TIRFM) allows imaging interface and near-surface processes selectively by avoiding the background noise with excellent surface sensitivity. Total internal reflection (TIR) occurs when light travels from a high refractive index medium $\left(\mathrm{n}_{1}\right)$ into a lower refractive index medium $\left(\mathrm{n}_{2}\right)$, when its angle of incidence $\left(\theta_{\mathrm{i}}\right)$ exceeds the critical angle $\left(\theta_{\mathrm{c}}\right), \theta_{c}=\sin ^{-1}\left(n_{2} / n_{1}\right)$. Under these conditions, light undergoes TIR off the interface into the high index medium. This TIR generates a standing electromagnetic field (evanescent wave) on the other side of the interface (in the lower index medium), of the same wavelength $(\lambda)$ as the incident light, with an intensity (I) that decays exponentially with distance from the interface $(\mathrm{z}), I=I_{0} e^{\left(-\frac{z}{d}\right)}$, where the characteristic exponential decay depth $\mathrm{d}$,

$$
d=\frac{\lambda}{4 \pi n_{2}}\left(\frac{\sin ^{2} \theta_{i}}{\sin ^{2} \theta_{c}}-1\right)^{-\frac{1}{2}}
$$

This evanescent wave is utilized in TIRFM to selectively excite fluorophores that are in close proximity (typically $<200 \mathrm{~nm}$ ) of the interface. This selective excitation results in a fluorescence image of the interface with no background fluorescence from the bulk providing excellent axial resolution ${ }^{1}$. When it comes to depth discrimination, evanescent field fluorescence performs up to ten times better than confocal light fluorescence microscopy ${ }^{2}$.

Several optical configurations of TIRFM have been developed. Broadly, they are classified into prism based ${ }^{3-5}$ and objective based techniques ${ }^{6,7}$. The prism-based systems are cumbersome to work with as the prism is coupled 
directly with the sample and this restricts the sample movement and often necessitates unconventional sample mounting schemes. It requires critical alignment to achieve TIR and prevents integration of other complementary techniques like atomic force microscopy $(\mathrm{AFM})^{8-11}$ or electrical conductance measurement, but provides the cleanest evanescent field. On the other hand, objective based TIRFM requires expensive high numerical aperture objectives to steer the illumination beam to achieve supercritical angles. Lower NA (low magnification) objectives that comprise the majority of fluorescence microscopy cannot be used for TIRFM. In addition, the evanescent field is polluted by the scattered light and autofluorescence from transmitting the excitation light through the objective thus reducing the signal-to-noise $(\mathrm{S} / \mathrm{N})$ ratio. Most of the TIRFM systems that are commercially available utilize laser sources that drive the cost up, especially for multi-wavelength imaging and they provide uneven illumination of the field due to the Gaussian intensity profile of the laser beam.

Here we describe a novel and inexpensive method of TIRFM wherein a high refractive index glass-sample interface is illuminated with high power light emitting diodes (LED), in an optical design that selects incident rays exceeding the critical angle. These rays undergo TIR resulting in the evanescent field on the sample side. The simple, open architecture allows integration of other complementary techniques like AFM, and has the potential to integrate other imaging modalities as well as lab-on-chip techniques thus providing a powerful research platform for biomedical applications.

\section{Results}

Microscopy setup. The device consists of a high refractive index cover glass (SF-11, RI: 1.78, ø $25 \mathrm{~mm}$ ), sandwiched between two thin flat black O-rings ( $4 \mathrm{~mm}$ rim width), placed under a stainless steel cylindrical tube (see Fig. 1 for details). This arrangement is placed concentrically in another stainless steel dish (ø $60 \mathrm{~mm}$ ) machined to have optical access from the bottom. Six high power LEDs mounted on a circular copper tube with thermal conductive epoxy were placed inside the outer dish. The entire setup was mounted on a Olympus IX-71 microscope equipped with $1024 \times$ 1024 EMCCD (Cascade II, Photometrics) or on a Nikon Eclipse TE2000-PFS system coupled with Bioscope SZ 1 AFM (Veeco Instruments, Santa Barbara, CA) equipped with $512 \times 512$ EMCCD (BT 2000, Diagnostic instruments) imaging systems.

Principle of operation. Light originating from the LEDs scatter diffusely at the waveguide surface, part of the light is trapped in the waveguide and undergoes refraction at all angles (Fig. 1a) at the glass-sample interface. For angles less than $\theta_{c}\left(\theta_{c}\right.$ being $48^{\circ}$, for the combination of 1.78 and 1.33 RIs), light refracts out into the other side of the cover glass and is absorbed by the black rubber seal. For angles greater than $48^{\circ}$, light undergoes total internal reflection within the cover glass and generates an evanescent field on either side of the cover glass interface. Scattered light or direct light from the source is prevented from reaching the sample by the inner cylindrical tube. For use with oil immersion objectives, the bottom surface of the coverslip is coated with a thin layer of a low index polymer (MY-133, RI 1.33) to prevent light scattering into the immersion oil.

Cell imaging: mapping connexin 43 hemichannels. To demonstrate the biological applications of this device for both live and fixed cell conditions, imaging was carried out on cells transfected with fluorescently tagged proteins. N2A cells transfected with connexin43-YFP (Cx43) grown on coverslips fixed with ice-cold methanol were imaged. Connexin43 is part of the gap junctional protein family associated with direct cell-cell (gap junctions) and cell-extracellular (hemichannels) communications. They are synthesized in the endoplasmic reticulum and transported to the cell membrane, which constitutes a cytoplasmic pool of $\mathrm{Cx} 43$, where they are assembled
(A)

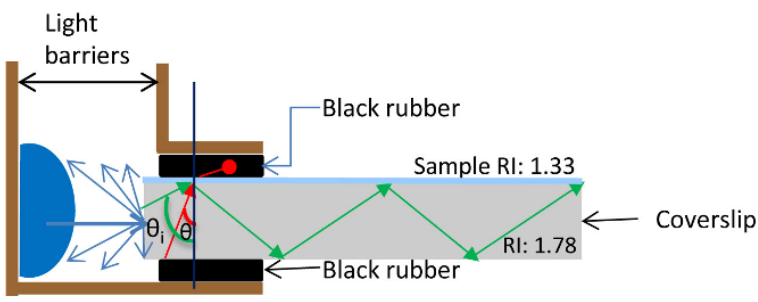

(B)

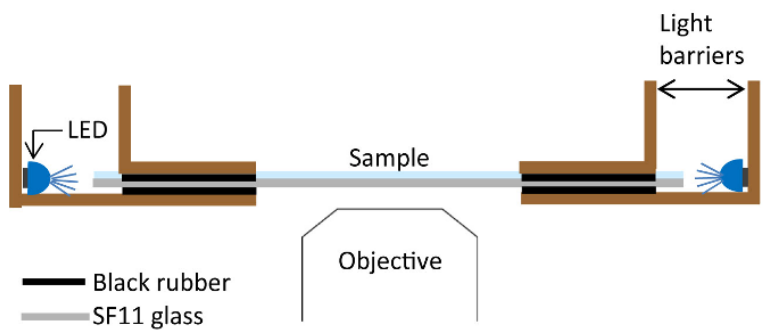

Figure 1 (A) Schematic of the LED based waveguide TIRF microscopy system and its principle of operation. Light from LED sources scatter diffusely at the waveguide surface, part of the light is trapped in the waveguide and undergoes refraction at all angles. For angles less than critical angle ' $\theta$ ' ( $\theta_{\mathrm{c}}$ being $48^{\circ}$, for the combination of 1.78 and 1.33 RIs), light refracts out into the other side of the cover glass and is absorbed by the black rubber. For angles greater than $48^{\circ}\left(\theta_{\mathrm{i}}\right)$, light undergoes total internal reflection within the cover glass and generates an evanescent field on either side of the cover glass interface. Scattered light or direct light from the source is prevented from reaching the sample by the inner cylindrical tube. (B) Schematic of the overall system design. A high index waveguide (SF 11 cover glass, R.I. 1.76, ø $25 \mathrm{~mm}$ ) sandwiched between thin black rubber sheets is mounted in the center of two concentrically arranged steel cylinders. The outer cylinder ( $\varnothing 60 \mathrm{~mm}$ ) machined to have optical access from the bottom for high magnification objectives. Six high power LED's mounted on a copper tube are placed inside the cylinder.

at the cell margins to carry out their cellular communication function.

A random field was chosen for imaging under both epi and TIRF modes (Fig. 2). The same field was imaged in aqueous media at different magnifications $10 \mathrm{x}, 20 \mathrm{x}$ and $40 \mathrm{x}$ water immersion. TIRF illumination provides excellent contrast (Fig. 2B, D, F) as it rejects the fluorescence contribution from the cytoplasmic pool of Cx43 and highlights only the membrane bound $\mathrm{Cx} 43$. On the other hand, epiillumination images suffer from lack of contrast due to fluorescence from cytoplasmic $\mathrm{Cx} 43$ (Fig. 2A, C, E). These images provide convincing evidence that the present technique is suitable for imaging structures in wide field of view even with low NA objectives.

Cell imaging: mapping paxillin in human mesenchymal stem cells. Imaging of live human mesenchymal stem cells transfected with paxillin-GFP (Fig. 2G,H), a focal adhesion-associated adaptor protein in the extracellular matrix that recruits diverse cytoskeleton and signaling proteins into a complex for cell signaling activity, showed the focal adhesion sites in the basal region clearly in TIRF mode (Fig. 2H). In contrast, in epi-illumination these sites are obscured by background (Fig. 2G). This demonstrates the method's suitability for live cell imaging. Similarly, a random field of methanol fixed Cx43-YFP was imaged with 100x oil immersion objective that once 

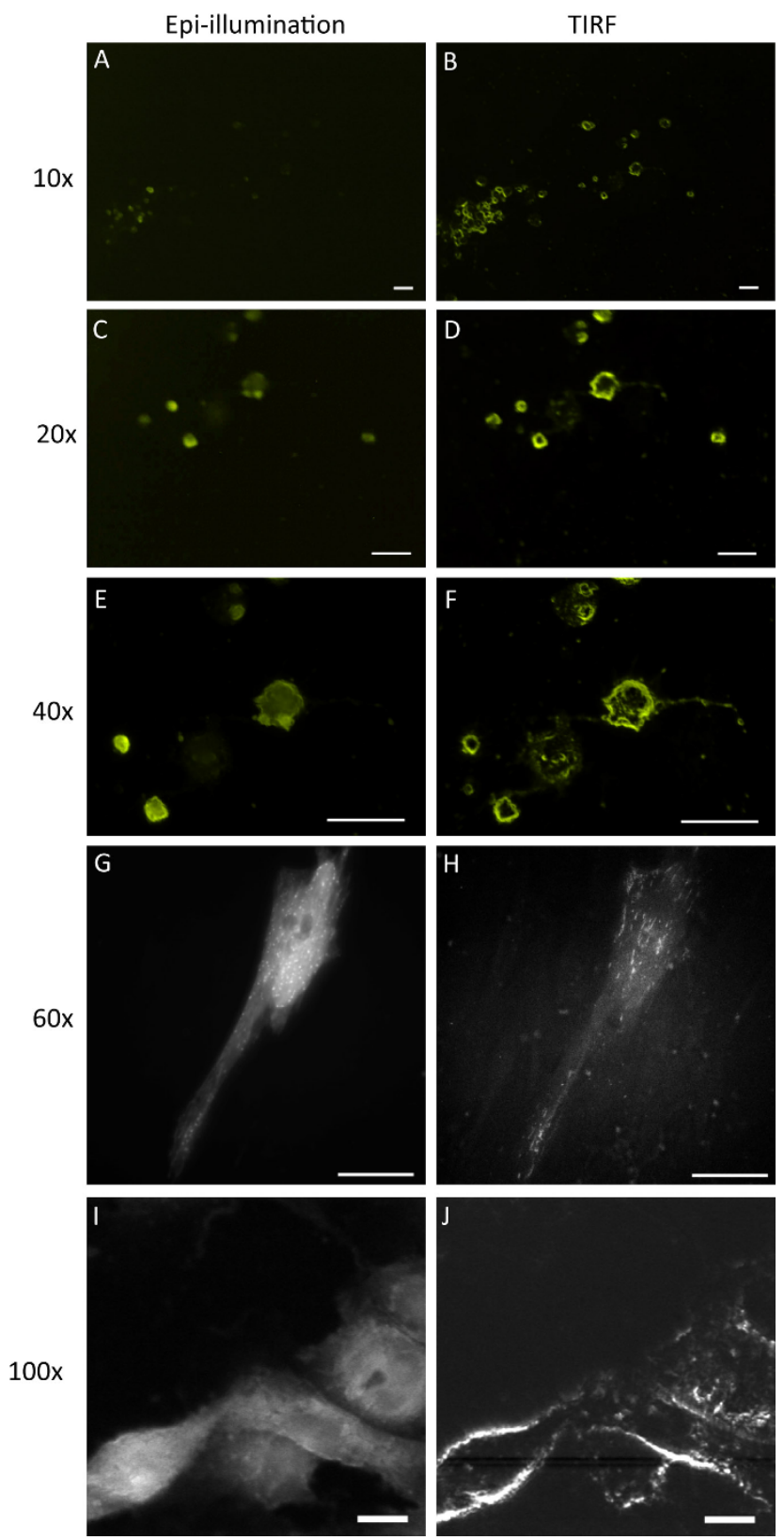

Figure $2 \mid$ TIRF imaging of cells with both low and high power objectives. Methanol fixed N2A cells transfected with Cx43-YFP were imaged with low power objectives $(10 \mathrm{x}-40 \mathrm{x}, \mathrm{A}-\mathrm{F})$ under both TIRF and epi-illumination modes. The TIRF image shows the distribution of $\mathrm{Cx} 43$ fluorescence at the margin of the cells while rejecting the background from cytoplasmic Cx43 that is evident in epi-illumination images. Live cell imaging of human mesenchymal stem cells transfected with paxillin-GFP was imaged with a 60x oil immersion objective $(\mathrm{G}, \mathrm{H})$. The TIRF panel shows the focal adhesion sites selectively while rejecting the background fluorescence from the cytoplasmic pool. 100x epi-illumination imaging of Cx43-YFP in methanol fixed cells (I) shows the $\mathrm{Cx} 43$ distribution across the entire thickness of the cell, while TIRF illumination (J) selectively shows Cx43 distributed at the periphery of the cells. Scale bar: $100 \mu \mathrm{m}$.

again demonstrates the surface sensitivity of TIRF microscopy (Fig. 2J).

Demonstration of TIRFM. In an another experiment, individual $200 \mathrm{~nm}$ diameter microspheres (Fluoresbrite ${ }^{\circledR} \mathrm{YG}$ ) were dispersed onto the cover glass and imaged against a fluorescent background of $10 \mathrm{mM}$ Lucifer Yellow dye solution (Fig. 3). The same field was imaged under TIRF and epi-fluorescence modes under identical imaging conditions. In epi-illumination, the contribution from the LY background was overwhelming and drastically reduced the image contrast (Fig. 3A). By comparison, under TIRF illumination, the background was nearly eliminated resulting in excellent image contrast (Fig. 3B). The intensity plot profile of the same region under both imaging conditions highlights the background contribution and reduction in image contrast in epifluorescence (Fig. 3A, inset) compared to TIRFM (Fig. 3B, inset). The base line of the intensity plot in epi fluorescence is elevated $(\sim 30,000)$ at least by fivefold compared to TIRF image $(\sim 6,000)$. Significantly, the base line is very even in TIRF mode suggesting that the field is evenly illuminated.

Characterization of evanescent field depth profile using AFM. The evanescent field depth profile was measured by attaching a 1 micron microsphere (Fluoresbrite ${ }^{\circledR} Y G$ ) to an AFM cantilevered tip (spring constant $200 \mathrm{~N} / \mathrm{m}$ ), which was then moved using a closed loop AFM scanner to precisely defined heights from the cover glass surface. Fluorescence images of the bead were captured at various heights, and the intensity as a function of tip-surface separation was measured using ImageJ software ${ }^{12}$. Such a method was described recently for calibration of the evanescent field in objective-based TIRF ${ }^{13,14}$. Figure 4 shows images of the fluorescent particle at different heights above the surface. A clear decrease in intensity is observed as a function of distance away from the surface. The evanescent field decay depth was determined empirically by calculating the height at which the evanescent field is $37 \%$ of its initial intensity at the surface $^{15}$. In our experiment, we obtained a decay depth of $200 \mathrm{~nm}$ using the fast TIRF component of the decay function $(210 \mathrm{~nm}$ when using the combined double exponential function), using an LED emitting at $455 \mathrm{~nm}$. The evanescent field depth is dependent on the wavelength of excitation light, its incidence angle, and the refractive indices of the glass coverslip and sample media ${ }^{13,14,16}$.

Multimodal compatibility of TIRFM. To demonstrate the open architecture and multimodal compatibility of our TIRFM, we carried out combined simultaneous AFM and TIRF microscopy on Cx43-YFP transfected cells. A region of the N2A cells was imaged using AFM prior to its removal by applying excessive force with the tip (force dissection) and simultaneous fluorescence images were acquired demonstrating the combined TIRF-AFM capability (Fig. 5). AFM height profile (Fig. 5F,G) provides excellent correlation with the evanescent depth profile of the TIRF system. The measured height of the neuronal process is about $200 \mathrm{~nm}$ (red arrows) as measured with AFM, while the cell body is saturated (Fig. 5F, $>200 \mathrm{~nm}$ from the surface), which is not registered in the TIRF image (Fig. 5B, blue arrows).

\section{Discussion}

Our LED powered, waveguide based TIRFM has several advantages over conventional prism or objective based systems. For example, i) in the objective based systems, special high numerical aperture objectives are used just to achieve super critical angles to realize TIR. In our system, since the illumination scheme is independent of microscope objective, any power objective $(10 \mathrm{x}$ to $100 \mathrm{x})$ could be used for imaging. ii) The entire waveguide is coupled to the light source which illuminates the entire surface with evanescent wave, a distinct advantage that solves the foremost drawback of laser based systems i.e., the variation in intensity of the evanescent field across the field of view caused by the Gaussian intensity distribution of the laser beam. iii) Uniform illumination of the entire field of view at once requires no sequential scanning of the sample (better temporal resolution). iv) In contrast to the prism based TIRF systems, the open architecture allows integration of other complementary modalities of investigation like AFM. v) It does not require any tedious alignment 

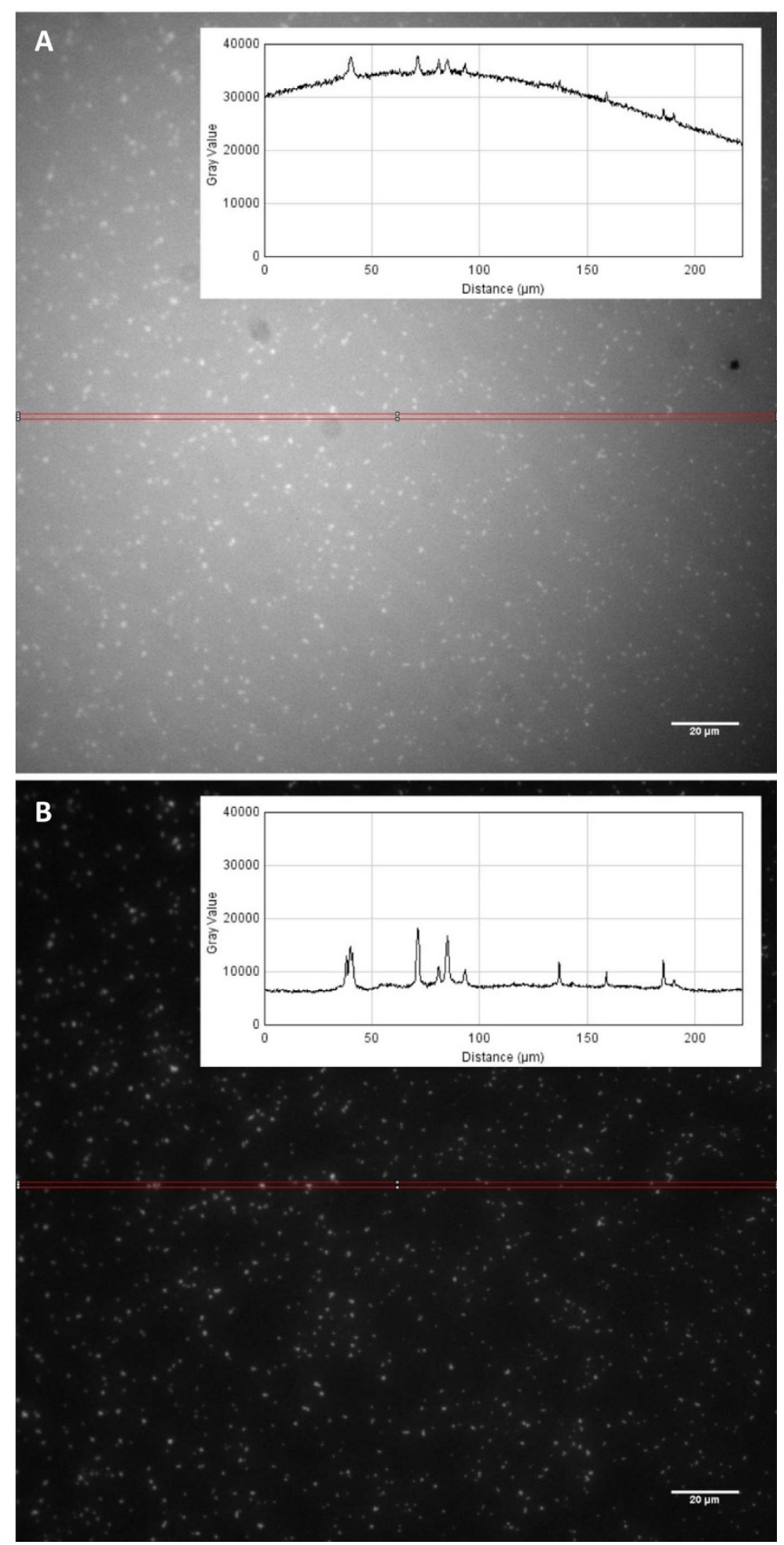

Figure 3 Demonstration of image contrast through comparative imaging of fluorescent beads against a background of Lucifer yellow dye under epiillumination (A) and TIRF (B) modes. Rejection of background fluorescence in the TIRF image results in better image contrast compared to the epiillumination. Corresponding intensity line plots (inset) across a narrow rectangular region of interest in the images shows the contribution of background fluorescence in epi-illumination, resulting in the upward shift of the baseline reducing the contrast. Scale bar is $20 \mu \mathrm{m}$.

procedure like the prism based TIRF and ( $\mathrm{f}$ ) above all, it could be an inexpensive add-on to any microscope system (upright/inverted) to achieve powerful TIRF imaging capability. In spite of the above limitations, laser based TIRF systems has some unique advantages. For example, they allow controlling the penetration depth of evanescent field by controlling the angle of incidence, and also allow polarization TIRF microscopy studies.
Recently, different versions of waveguide TIRFM systems have been reported ${ }^{17-21}$. Grandin et al. ${ }^{20}$, coupled a laser source into a planar waveguide via optical grating embedded on it at a specific angle with a goniometer. This technique requires perfect alignment as efficient incoupling happens only at a specific angle of incidence that is dependent on the wavelength, the grating properties, as well as the refractive indices of the substrate, the waveguide and the media 

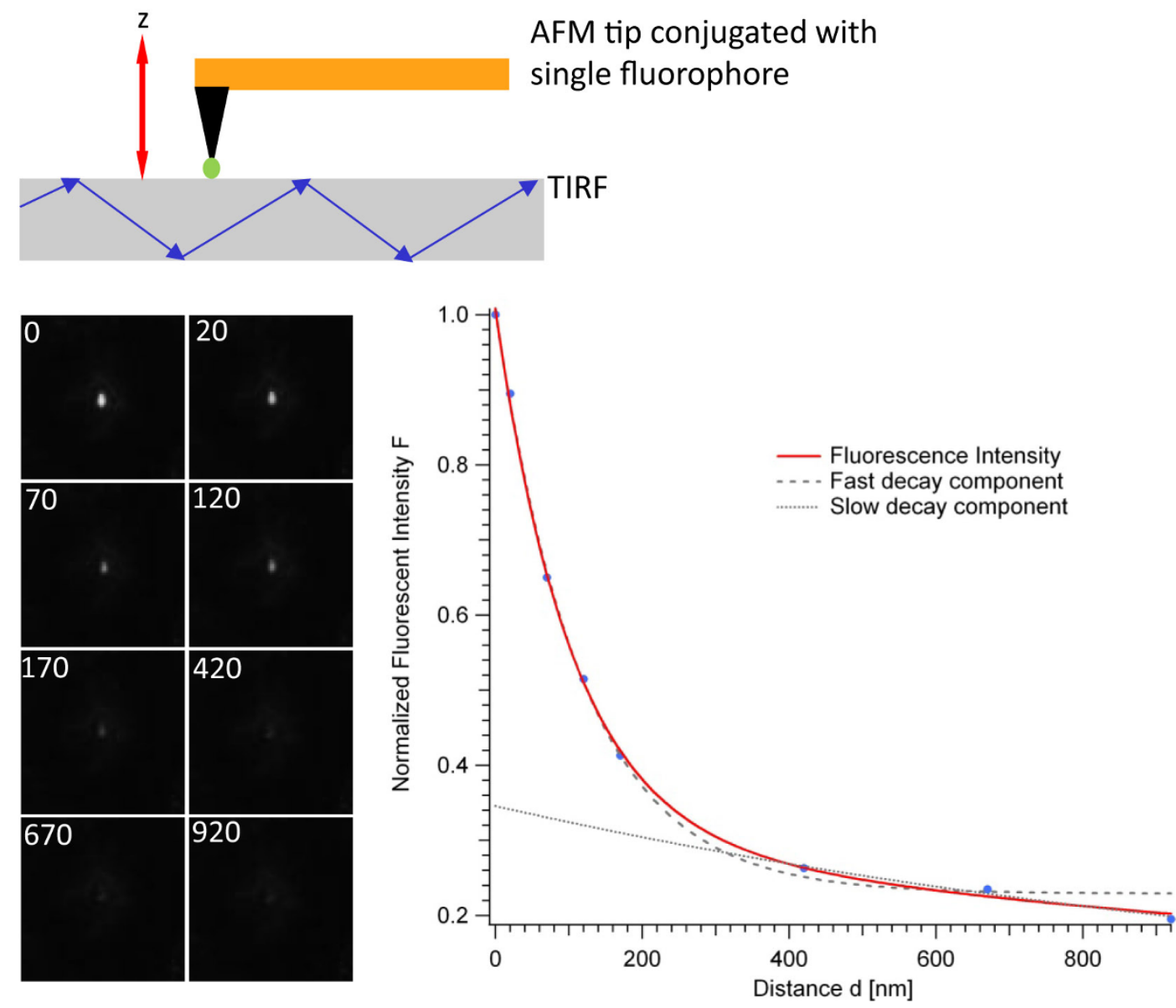

Figure $4 \mid$ Measurement of evanescent field depth using AFM. A fluorescent bead immobilized on the tip of a cantilever $(k=200 \mathrm{~N} / \mathrm{m})$ was moved away from the surface at defined intervals using a high precision AFM scanner. Using force curve motion of the scanner, and halting the movement for 5 seconds both at the top and bottom of the force curve movement, fluorescence images were collected at different positions of z-travel of the AFM scanner using different $Z$ scan sizes in the force curves. An $80 \mathrm{~nm}$ trigger was used to determine the exact point at which tip-surface separation occurred. Fluorescence intensities were normalized with respect to the surface fluorescence. The plot shows the characteristic exponential decay of the evanescent field.

above the grating. Though it offers extreme sensitivity of target detection, multi-wavelength image with wide field of view capabilities, the image quality obtained using this method was limited by uneven illumination resulting from imperfections in the in-coupling grating $^{18}$. Hassanzadeh et al. ${ }^{17}$, created a high refractive index waveguide by exchanging low polarizability ions with high polarizability ones and coupled a laser source through a sub-micron grating on one end of the waveguide to achieve evanescent field fluorescence microscopy. Agnarsson et al. ${ }^{18}$, used a symmetric waveguide structure to achieve waveguide excitation fluorescence microscopy. This method provides flexibility of evanescence excitation in addition to the other advantages of the waveguide based TIRFM. But all these methods require extensive clean room fabrication procedures, alignment and expertise in optics. Our approach requires only a commercially available cover glass and a single low cost attachment to a common microscope. Chronis et al. ${ }^{21}$, have developed a lab-on-chip system where a microfabricated cavity filled with optical polymer and a side wall mirror design couples a laser beam to a glass-sample interface at greater than critical angle achieving evanescent wave excitation of fluorescent samples without any tedious alignment requirements. This system is also integrated with microfluidics for rapid analysis of samples. Imaging is accomplished by dry lenses in transmittance mode which precludes interfacing with other investigating techniques. Asanov et al. ${ }^{19}$, developed a proprietary system wherein the source light was coupled into a rectangular coverslip physically through a beam conditioner that injects light through a prism at one end of the coverslip. An optical trap to extinguish shallow angle illumination is placed at the other end on the surface of the coverslip. Our optical design, on the other hand, is simple; it does not use any prism, the incident rays below the critical angle are filtered out as they refract to the other side of the coverslip and absorbed by the black rubber. The absence of the prism and other constraints in our open design architecture as well as its ability to image in reflectance mode allows easy integration of our system with other complementary investigative modalities like electrophysiology, AFM etc. Such a combined system will be very useful for probing structure-function activity of plasma membrane macromolecular structures (including channels, receptors, lipids, glycoproteins, etc.) either in purified membranes or in the basal cell membrane for real-time clustering, trafficking, and their interactions with extracellular matrix that defines the cell activity. Moreover, it will also help define the signal transduction pathways that include multilayered cytoskeletal interactions and related biomechanical components (using AFM and TIRFM) as would occur in response to the interaction of external stimuli with the cell plasma membrane and the extracellular matrix (TIRFM).

The evanescent field decay depth was determined empirically by computing the depth at which the evanescent wave is $37 \%$ of its initial intensity at the surface ${ }^{15}$. In our experiment using AFM-TIRF system, we obtained a decay depth of $200 \mathrm{~nm}$ using the fast TIRF 

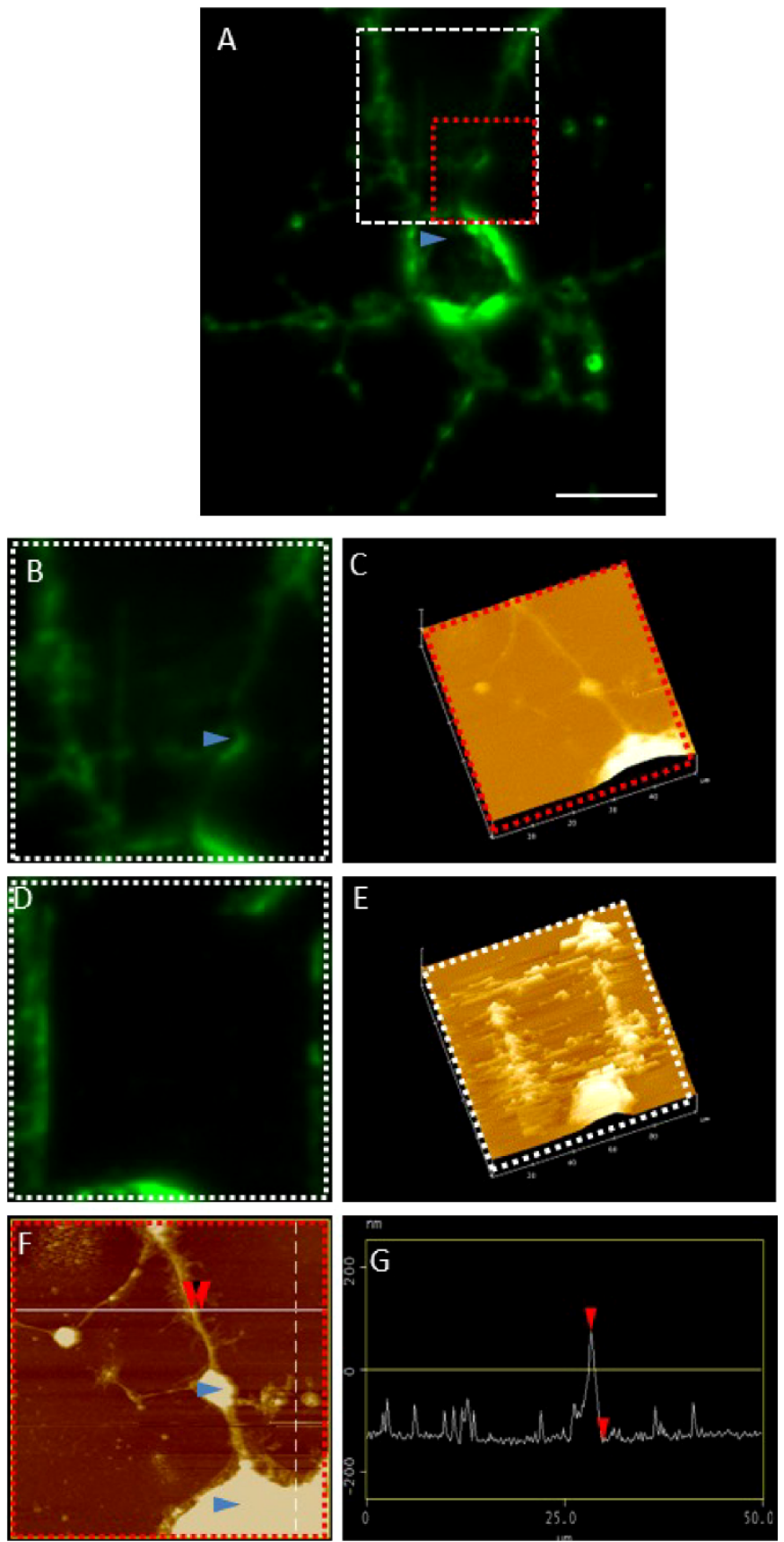

Figure 5 | Simultaneous combined AFM and TIRF imaging of cells. Simultaneous AFM and TIRF imaging was carried out on methanol fixed N2A cells transfected with Cx43-YFP. (A) TIRF image of a cell before it was force dissected using the AFM tip. The white and red rectangular boxes indicate region of interest (ROI) zoomed in for AFM imaging. (B, C) TIRF and AFM images respectively of the ROI before force dissection, $(D, E)$ are corresponding images after force dissection. (F, G) The height of the neuronal process to be $\sim 200 \mathrm{~nm}$ (red arrows) which is within the evanescence field of excitation; cell bodies that are $>200 \mathrm{~nm}$, are saturated in AFM images, are not registered in TIRF images (blue arrows).

component of the decay function $(210 \mathrm{~nm}$ when using the combined double exponential function). This is well within the range of values reported for lens-based TIRF systems that describe the two-component decay function ${ }^{13,15,16}$. Further, the origin of the slow decay component is attributed to the scattering components (e.g., objective, index mismatch between sample and its medium, inefficiency of collection due to far field surface reflections). In our device at
$455 \mathrm{~nm}$ excitation, the slow scattering component does not become dominant until the tip is more than $300 \mathrm{~nm}$ away from the surface.

Due to the difference in design of our TIRFM from the existing TIRFM systems, a head-to-head comparison of our system with a conventional TIRFM system with same samples is beyond the scope of this work. We have compared our results with published work on Cx43 using through objective TIRFM ${ }^{22}$, our results are consistent with the published work.

A distinct advantage of this system is in achieving excellent surface sensitivity using low NA dry/water objectives $(5 x-40 x)$. A potential application of this could be in imaging dynamic events over a large field of view. For instance, imaging calcium waves in a network of neurons, axonal transport across long neurons, imaging fluorescently tagged rapidly moving cells or C. elegans etc. Further, functionalized waveguides could be employed in a variety of applications including molecular diagnostics, bio-molecular kinetics, surface chemistry applications etc. Because of its open architecture, this system could be used on upright microscopes as well. However, further improvements in optical power of the excitation are needed to extend its application to single molecule studies.

In conclusion, we have successfully demonstrated a novel inexpensive TIRF device that utilizes a high index waveguide illuminated by LEDs to achieve TIRFM that can be easily used as an add-on device on any standard optical microscope to gain TIRFM functionality for the study of complex interfacial interactions, ranging from the study of membrane proteins (such as ion channels, membrane transport proteins, receptors, receptor-ligand interactions), visualization of the cell-extracellular matrix (ECM) interactions, tracking of secretory granules and protein trafficking ${ }^{23-28}$ over a wide field of view using low NA objectives.

\section{Methods}

SF-11, a high index (1.78), $25 \mathrm{~mm}$ diameter and \#1.5 thick glass cover glass, was purchased from V.A. Optical Labs, San Anselmo, CA. Philips Luxeon K2 high power light emitting diodes (LED) of different wavelengths (455 [475 mW@1A], 470 [21 lm@1A], 505 [80 lm@1A], 530 [1001m@1A]) were purchased from Future Electronics, Canada. Thermal conductive epoxy was obtained from Epoxies, etc., (Cranston, RI). Low refractive index (1.33) cladding material MY-133 was obtained from MY-Polymers Ltd., Rehovot, Israel. Fluoresbrite ${ }^{\circledR}$ YG 0.2 and $0.05 \mu \mathrm{m}$ microspheres (Ex/Em: 441/486 nm) were purchased from Polysciences Inc. Lucifer Yellow (LY, Ex/Em: 428/536 nm) was obtained from Invitrogen (Carlsbad, CA). N2A cells, EMEM, and FBS were obtained from American Type Culture Collection (Manassas, VA). Lipofectamine 2000 and Opti-MEM ${ }^{\circledR}$ were obtained from Invitrogen (Carlsbad, CA).

Evanescent field profile measurement with AFM. The evanescent field depth was measured by attaching a 1 micron diameter Fluoresbrite ${ }^{\circledR}$ fluorescent microsphere (Polysciences, Excitation/Emission: 441/530 nm) to an AFM tip (cantilever spring constant $200 \mathrm{~N} / \mathrm{m}$ ) by lowering the tip first on a thin film of nearly dry epoxy followed by lowering the tip on fluorescent particles that were freshly spread on a glass slide. The AFM scanner (Bioscope SZ 1) was inserted into the top access of the device and force curves were initiated ${ }^{29}$. During force curve motion of the AFM scanner, the tip was halted for 5 seconds both at the lower and upper end of the force curve to allow for fluorescence image acquisition. Using an $80 \mathrm{~nm}$ trigger (pushing the cantilever down $80 \mathrm{~nm}$ upon surface contact in the down motion) and an extremely stiff cantilever, the exact start point of tip-surface separation could be established by offsetting the upwards motion $80 \mathrm{~nm}$. The high spring constant prevented the tip from sticking to the surface in the upwards motion during a force curve. Fluorescence image acquisition was done using an EMCCD camera (SPOT BOOST, $512 \times 512$ pixels, and $900 \mathrm{~ms}$ acquisition time). The LED wavelength used was $455 \mathrm{~nm}$. We then measured the intensity of light emitted from the fluorescent particle as a function of tip-surface separation using ImageJ software ${ }^{12}$.

Cell culture. N2A cells, a mouse neuroblastoma cell line, obtained from ATCC was maintained in Eagles minimum essential medium (EMEM) supplemented with $10 \%$ fetal bovine serum in a humidified sterile cell culture chamber at $37^{\circ} \mathrm{C}$ and $5 \%$ CO2. For the Cx43-YFP transfection, cells grown on sterile coverslips up to $70 \%$ confluence and transfected with Cx43-YFP using Lipofectamine 2000 as per the manufacturer's recommended protocol with slight modifications. Briefly, cells washed free of serum proteins in PBS, were treated with $500 \mu \mathrm{l}$ of Lipofectamine - plasmid mixture at 3:1 ratio. Both Lipofectamine and Cx43-YFP $(1 \mu \mathrm{g})$ were diluted in Opti-MEM I before adding it to the cells. Media was exchanged after $6 \mathrm{hrs}$ with regular media and cells were imaged after another $12 \mathrm{hrs}$ of incubation. Cells were fixed in ice-cold methanol and imaged. Live human mesenchymal stem cells 
transfected with paxillin-GFP, were a generous gift from Dr. Varghese's lab (Dept. of Bioengineering, UCSD).

Optical imaging. Most of the imaging was carried out on inverted optical microscope (Olympus IX71, Japan) equipped with $75 \mathrm{~W}$ Xenon light source, high contrast-zero pixel shift filter cubes (DAPI/FITC/TRITC/TxRed/YFP, Semrock, NY), Olympus objectives: $10 \times($ UMPlanFl, NA 0.3), $20 \times($ UMPlanFl, NA 0.46), $40 \times$ W (UApo, NA $1.15), 60 \times \mathrm{O}$ (UPlanSApo, NA 1.35) and $100 \times \mathrm{O}$ (UPlanSApo, NA 1.4), XYZ motorized stage (Prior ProScan II) and ImageEM-1k EMCCD (Hamamatsu, Japan). AFM-TIRFM experiments were carried out on a TE2000-PFS (Nikon, NY) optical microscope combined with Bioscope SZ1 closed loop scanner (Veeco Instruments, Santa Barbara) connected to a $512 \times 512$ EMCCD (SPOT Boost, Diagnostic Instruments).

1. Axelrod, D. Cell-substrate contacts illuminated by total internal reflection fluorescence. The Journal of Cell Biology 89, 141-145 (1981).

2. Steyer, J. A. \& Almers, W. A real-time view of life within $100 \mathrm{~nm}$ of the plasma membrane. Nat Rev Mol Cell Bio 2, 268-275 (2001).

3. Axelrod, D., Thompson, N. L. \& Burghardt, T. P. Total Internal-Reflection Fluorescent Microscopy. J Microsc-Oxford 129, 19-28 (1983).

4. Axelrod, D., Burghardt, T. P. \& Thompson, N. L. Total Internal-Reflection Fluorescence. Annu Rev Biophys Bio 13, 247-268 (1984).

5. Burghardt, T. P., Hipp, A. D. \& Ajtai, K. Around-the-objective total internal reflection fluorescence microscopy. Appl Optics 48, 6120-6131 (2009).

6. Stout, A. L. \& Axelrod, D. Evanescent Field Excitation of Fluorescence by Epi-Illumination Microscopy. Appl Optics 28, 5237-5242 (1989).

7. Axelrod, D. Total internal reflection fluorescence microscopy in cell biology. Traffic 2, 764-774 (2001).

8. Hansma, P. K. et al. A New, Optical-Lever Based Atomic-Force Microscope. J Appl Phys 76, 796-799 (1994)

9. Lal, R. \& John, S. A. Biological Applications of Atomic-Force Microscopy. Am J Physiol 266, C1-\& (1994).

10. Ramachandran, S. \& Lal, R. Scope of atomic force microscopy in the advancement of nanomedicine. Indian Journal of Experimental Biology 48, 1020-1036 (2010).

11. Lal, R. \& Yu, L. Atomic-Force Microscopy of Cloned Nicotinic AcetylcholineReceptor Expressed in Xenopus-Oocytes. Proceedings of the National Academy of Sciences of the United States of America 90, 7280-7284 (1993).

12. Rasband, W. S. \& Bright, D. S. Nih Image - a Public Domain Image-Processing Program for the Macintosh. Microbeam Anal 4, 137-149 (1995).

13. Oreopoulos, J. \& Yip, C. M. Combined scanning probe and total internal reflection fluorescence microscopy. Methods 46, 2-10 (2008).

14. Sarkar, A., Robertson, R. B. \& Fernandez, J. M. Simultaneous atomic force microscope and fluorescence measurements of protein unfolding using a calibrated evanescent wave. Proceedings of the National Academy of Sciences of the United States of America 101, 12882-12886 (2004).

15. Keyel, P. A., Watkins, S. C. \& Traub, L. M. Endocytic adaptor molecules reveal an endosomal population of clathrin by total internal reflection fluorescence microscopy. J Biol Chem 279, 13190-13204 (2004).

16. Mattheyses, A. \& Axelrod, D. Direct measurement of evanescent field profile and depth in TIRF microscopy. Biophys J 86, 320a-320a (2004).

17. Abdollah Hassanzadeh, M. N., Silvia Mittler, Souzan Armstrong \& Jeff Dixon, a. U. L. Waveguide evanescent field fluorescence microscopy: Thin film fluorescence intensities and its application in cell biology. Applied Physics Letters 92, 233503 (2008)

18. Agnarsson, B., Ingthorsson, S., Gudjonsson, T. \& Leosson, K. Evanescent-wave fluorescence microscopy using symmetric planar waveguides. Opt Express 17, 5075-5082 (2009)
19. Asanov, A., Zepeda, A. \& Vaca, L. A novel form of Total Internal Reflection Fluorescence Microscopy (LG-TIRFM) reveals different and independent lipid raft domains in living cells. Biochim Biophys Acta 1801, 147-155 (2010).

20. Grandin, H. M., Städler, B., Textor, M. \& Vörös, J. Waveguide excitation fluorescence microscopy: A new tool for sensing and imaging the biointerface. Biosensors and Bioelectronics 21, 1476-1482 (2006).

21. Chronis, N. \& Lee, L. P. Total internal reflection-based biochip utilizing a polymer-filled cavity with a micromirror sidewall. Lab on a Chip 4, 125-130 (2004).

22. Shaw, R. M. et al. Microtubule plus-end-tracking proteins target gap junctions directly from the cell interior to adherens junctions. Cell 128, 547-560 (2007).

23. Moerner, W. E. \& Orrit, M. Illuminating single molecules in condensed matter. Science 283, 1670-+ (1999).

24. Wiegand, U. K. et al. Exocytosis studies in a chromaffin cell-free system - Imaging of single-vesicle exocytosis in a chromaffin cell-free system using total internal reflection fluorescence microscopy. Chromaffin Cell: Trnsmitter Biosynthesis, Storage, Release, Actions, and Informatics 971, 257-261 (2002).

25. Mica, O. I. et al. TIRF imaging of docking and fusion of single insulin granule motion in primary rat pancreatic beta-cells: different behaviour of granule motion between normal and Goto-Kakizaki diabetic rat beta-cells. Biochem J 381, 13-18 (2004).

26. Krementsova, E. B., Hodges, A. R., Lu, H. L. \& Trybus, K. M. Processivity of chimeric class V myosins. J Biol Chem 281, 6079-6086 (2006).

27. Kumari, S. et al. Nicotinic acetylcholine receptor is internalized via a Racdependent, dynamin-independent endocytic pathway. J Cell Biol 181, 1179-1193 (2008).

28. Saffarian, S. \& Kirchhausen, T. Differential evanescence nanometry: Live-cell fluorescence measurements with 10-nm axial resolution on the plasma membrane. Biophys J 94, 2333-2342 (2008).

29. Almqvist, N. et al. Elasticity and adhesion force mapping reveals real-time clustering of growth factor receptors and associated changes in local cellular rheological properties. Biophys J 86, 1753-1762 (2004).

\section{Acknowledgements}

The authors acknowledge Davis Carlin, Drs. Clark Hyde, Preston B. Landon and Fernando T. Arce for helpful discussions. We thankfully acknowledge Dr. Shyni Varghese of Bioengineering department, UCSD, for the generous gift of paxillin transfected mesenchymal stem cells for imaging. This work was supported by grants from NIH DA025296; DA024871 to R

\section{Author contributions}

S.R., D.A.C., A.P.Q. and R.L. conceived the idea and prepared the manuscript; S.R., D.A.C., A.P.Q. performed the experiments and analyzed the data

\section{Additional information}

Competing financial interests: The authors declare no competing financial interests.

How to cite this article: Ramachandran, S., Cohen, D.A., Quist, A.P. \& Lal, R. High performance, LED powered, waveguide based total internal reflection microscopy. Sci. Rep. 3, 2133; DOI:10.1038/srep02133 (2013)

(1) (-) This work is licensed under a Creative Commons AttributionBY NC ND NonCommercial-NoDerivs 3.0 Unported license. To view a copy of this license, visit http://creativecommons.org/licenses/by-nc-nd/3.0 